\title{
openheart Applying the ordinal model of atherosclerosis to imaging science: a brief review
}

\author{
Jacob W Groenendyk, Nehal N Mehta
}

To cite: Groenendyk JW, Mehta NN. Applying the ordinal model of atherosclerosis to imaging science: a brief review. Open Heart 2018;5:e000861. doi:10.1136/ openhrt-2018-000861

Received 18 May 2018 Revised 30 May 2018 Accepted 3 July 2018
Check for updates

\section{(C) Author(s) (or their} employer(s)) 2018. Re-use permitted under CC BY-NC. No commercial re-use. See rights and permissions. Published by BMJ.

National Heart, Lung, and Blood Institute, National Institutes of Health, Bethesda, Maryland, USA

Correspondence to Dr Nehal N Mehta; nehal. mehta@nih.gov

\section{ABSTRACT}

Atherogenesis has been well demonstrated to proceed in an ordinal fashion. Imaging technologies have advanced substantially in recent decades, enabling early detection of atherosclerosis. Some modalities, such as coronary CT, have seen broad clinical adaptation. In contrast, others, such as flow-mediated dilatation, remain predominantly research-based. 0ptimal and appropriate usage of these technologies remains an area of active investigation. We hypothesise that investigators ought to consider which stage of atherosclerosis is under investigation when choosing imaging modalities. Additionally, when assessing the efficacy of a particular treatment, some imaging modalities may be more appropriate than others. We review the most important available imaging modalities and suggest stages at which each may or may not be well used. Conceptual application of the classic stages of atherosclerosis model to the variety of modern imaging modalities available will result in more effective investigation and treatment of cardiovascular disease.

\section{INTRODUCTION}

Cardiovascular disease (CVD) is the leading cause of mortality worldwide; in the USA, coronary disease is implicated in one in six deaths. ${ }^{1}$ Treatment and prevention of CVD is a high priority both domestically and worldwide. Contributors to CVD have historically been understood to include the 'traditional' risk factors, such as family history, obesity, tobacco use, diabetes, dyslipidaemia and hypertensive disease. ${ }^{2}$ In recent years, a number of clinical trials such as Justification for the Use of Statins in Prevention: an Intervention Trial Evaluating Rosuvastatin (JUPITER), Pravastatin or Atorvastatin Evaluation and Infection Therapy-Thrombolysis in Myocardial Infarction 22 (PROVE IT-TIMI 22), and Canakinumab Antiinflammatory Thrombosis Outcome Study (CANTOS) have shed light on inflammatory contributions to CVD. ${ }^{3-5}$ Understanding and interest in the processes that contribute to atherosclerosis, and thus CVD, have continued to progress in conjunction with the development of novel imaging technologies that allow for earlier and finer characterisation of the atherogenic process.

Atherogenesis, the process of plaque build-up in the arterial wall, is thought to proceed in an ordinal fashion ${ }^{1}$ (figure 1). The first stage is characterised by qualitative changes to endothelial cells lining the vessel lumen and the subsequent expression of proadhesive molecules that recruit immune cells in response to stimuli such as lipid build-up or hypertensive pressure. Recruited leucocytes then initiate residence in the tunica intima. Monocyte-derived macrophages take up lipoprotein particles to become foam cells. Smooth muscle cells also traverse from the tunica media to the tunica intima. These cells produce extracellular matrix components such as elastin and collagen, which form a cap that covers the plaque. Plaque growth can compromise blood flow through the vessel lumen, resulting in ischaemia and symptoms of impaired perfusion such as stable angina or peripheral arterial disease. Alternatively, plaque rupture can occur, resulting in infarction. ${ }^{2}$ Plaque rupture is responsible for the majority of myocardial infarction (MI), with estimates ranging from $60 \%$ to $70 \%$, the majority of these plaques being non-calcified. ${ }^{34}$

Primary prevention, or preventative measures that occur before the relevant event occurs, offers great opportunity for reducing both human and economic burdens of CVD. ${ }^{56}$ Currently, treatment decisions are based on demographics and clinically obtained data, such as blood pressure, lipid levels and current treatments. ${ }^{7}$ Novel preventative strategies for CVD are continually being developed; imaging data may be helpful in determining which patients are at the highest risk or which patients may benefit most from a particular treatment. Information on risk and response, in turn, can be used to appropriately titrate treatment to minimise the burden of unnecessary costs and side effects. 


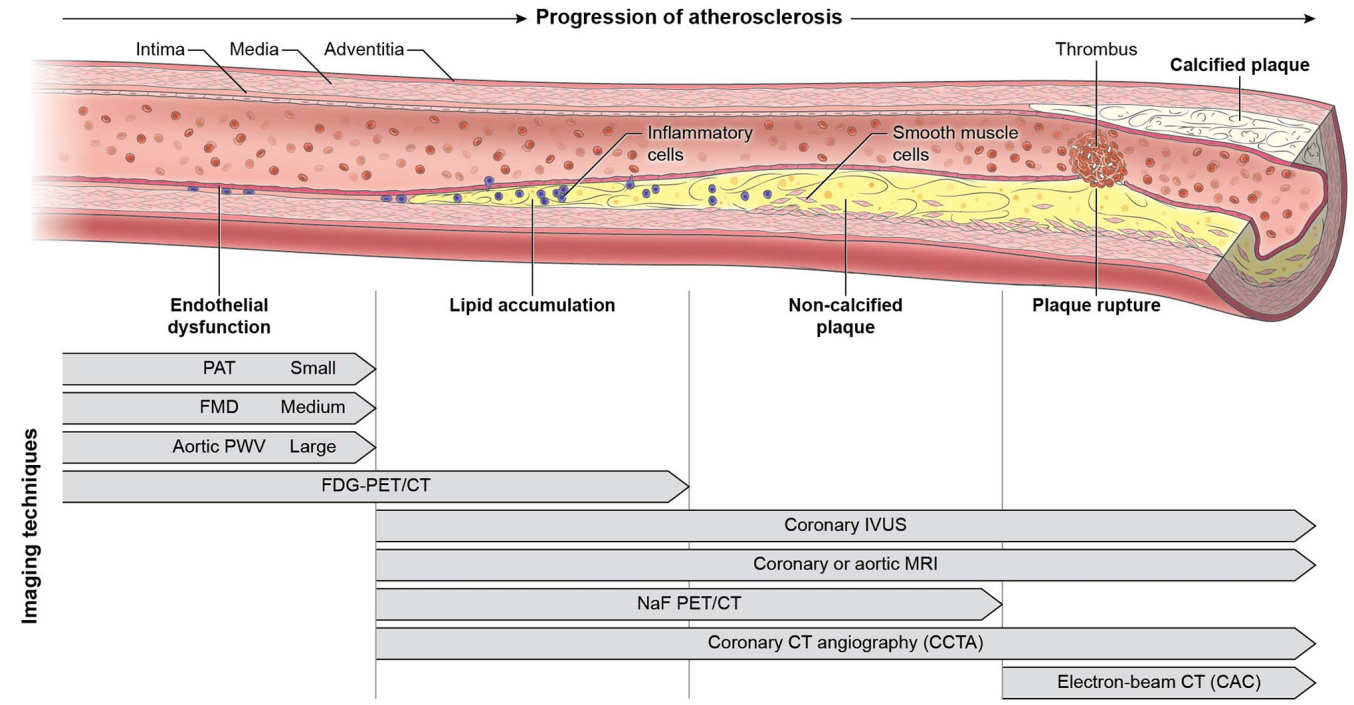

Figure 1 The progression of atherosclerosis by different imaging modalities. This figure depicts how atherosclerosis begins with endothelial dysfunction, followed by expression of proadhesive molecules that recruit monocytes in response to stimuli such as hypertensive pressure or lipid build-up. These monocytes then take up lipids to become foam cells. Additionally, smooth muscle cells migrate from the tunica media to the tunica intima, where they produce elastin and collagen, which create a cap that covers the plaque. Plaque growth can compromise blood flow to distal regions, resulting in stable angina or peripheral arterial disease. Alternatively, erosion of the fibrous cap can expose prothrombotic mediators, resulting in clot formation and infarction, either in the myocardium or the brain. Imaging modalities for these stages should be selected based on the physiological changes expected at each stage. CAC, coronary artery calcium; FDG-PET, 18-fluorodeoxyglucose positron emission tomography; FMD, flow-mediated dilatation; NaF, sodium fluoride; IVUS, intravascular ultrasound; PAT, peripheral arterial tonometry, small arteries; PWV, pulse wave velocity, large arteries.

While multiple reviews on the imaging of atherosclerosis have been published, none have placed special emphasis on tailored imaging modalities with the stages of atherosclerosis model. $^{8-12}$ This review summarises the various imaging modalities currently in use, both clinically and in the research setting, and proposes a paradigm in which different modalities have different levels of usefulness for different stages of atherogenesis. Further research using large populations will be needed to validate this hypothesis; it is possible that appropriately choosing an imaging modality is critical to the success or failure of investigation, both interventional and observational. Herein, we have grouped imaging modalities into one of two categories: functional or anatomical. We acknowledge not all modalities will fit this structure perfectly. For example, aortic MRI can be used to obtain both structural (i.e., aortic wall thickness or lumen area) and functional (i.e., aortic distensibility) parameters.

\section{EARLY ATHEROSCLEROSIS: ENDOTHELIAL FUNCTION TESTING AND AORTIC CHARACTERISATION}

Measurement of endothelial dysfunction can be a helpful assessment of cardiovascular risk. Flow-mediated dilatation (FMD), which uses ultrasound to measure the brachial artery before and after flow restriction induced via cuff occlusion, dates to late 1992 and quantifies the change in diameter associated with nitric oxide release. Presently, FMD is the most commonly used method of clinical assessment of endothelial dysfunction in a research setting. ${ }^{13-15}$ However, FMD values obtained can vary significantly by site and operator. ${ }^{16}$ Peripheral arterial tonometry (PAT), which operates using similar principles but uses a finger clip device to monitor vasodilation rather than Doppler ultrasound, may be less susceptible to user variability. ${ }^{17}$ Increases in both FMD and PAT have been demonstrated to predict decrease in cardiovascular events. ${ }^{14}$

18-fluorodeoxyglucose positron emission tomography (FDG-PET) imaging was initially developed in the 1970s. When combined with CT imaging, it can detect locations of increased metabolic uptake of radiolabelled glucose. The signal from FDG-PET may be greatest early in the atherosclerotic process, when foam cells are active. ${ }^{18}$ However, FDG-PET can also detect early endothelial inflammatory changes. A small study demonstrated correlation between increased PET/CT uptake and histological evidence of increased macrophage activity at suspicious lesions in the coronary arteries after MI. ${ }^{19}$ Additionally, patients randomised to high-dose statins have decreased vessel FDG uptake compared with patients who receive low-dose statin therapy ${ }^{20}$ On retrospective analysis, arterial target to background ratio, a measure of FDG uptake in the arterial wall, improves the predictive accuracy of cardiovascular events beyond the Framingham risk score. ${ }^{21}$

Pulse wave velocity (PWV), the rate at which arterial pulse moves through the vasculature, can be measured non-invasively via a variety of methods; the most common include tonometry and cine MRI. This value is used as a proxy for stiffness, as the pulse wave moves more 
rapidly through stiffer vessels. Furthermore, PWV can be measured in multiple locations, such as the thoracic aorta, abdominal aorta, iliac artery and the femoral artery. Carotid-femoral PWV via tonometry is most commonly measured clinically; Ankle-Brachial Index (ABI) may also be used. ${ }^{22}$ When PWV is measured transcutaneously, operators must measure distance precisely, as even small differences in measurement can alter the value obtained. Data from the Framingham study show significant increase in PWV with age. ${ }^{23}$ Multiple studies have demonstrated that aortic PWV is predictive of CVD, even after adjustment for traditional cardiovascular risk. ${ }^{24-26}$ While PWV measures global stiffness of a section of the vasculature, aortic distensibility can be measured in a specific location via cine MRI. Decreased distensibility in the ascending aorta correlated with increased risk of cardiovascular events in the Multi-Ethnic Study of Atherosclerosis (MESA) study. ${ }^{27}$ Both PWV and distensibility likely measure a relatively later manifestation than endothelial cell activation; increases in PWV and distensibility likely occur concurrently with plaque build-up and smooth muscle cell accumulation, although further investigation in this area is needed to better understand the pathophysiology.

$\mathrm{ABI}$, the ratio of systolic blood pressure in the upper arm compared with that in the lower leg, has long been used in the diagnosis of peripheral arterial disease. Diagnosis of peripheral arterial disease via ABI correlates with an approximately fivefold increased risk of 10 -year cardiovascular mortality. ${ }^{28}$ While $\mathrm{ABI}$ is not strictly an imaging technique, it is important to consider as a commonly used functional measure of atherosclerosis, most commonly in the setting of diagnostic work-up for claudication.

\section{Summary}

These functional imaging methodologies, with the notable exception of ABI, likely represent the development of early changes to the endothelium and intima, and thus associated distensibility property changes. These modalities, which target endothelial activation or smooth muscle cell proliferation, would be best used for detecting early changes in atherosclerotic disease.

\section{ANATOMICAL EVALUATION OF ATHEROSCLEROSIS}

Thickness of the aortic wall can be measured via MRI and is thought to represent preclinical CVD after the stage of endothelial dysfunction, inflammation and stiffness has occurred. This may represent a later stage of atherosclerosis than other imaging modalities, such as FDG-PET, that focus primarily on inflammation. Older age, male gender and hypertension are predictive of increased aortic wall thickness. ${ }^{29}$ While it is established that arterial thickness can be predictive for cardiovascular events, the prognostic value of aortic thickness in the thorax is presently unknown. However, data from the Dallas Heart Study showed a prospective increase in the risk of composite cardiovascular events (HR 1.28 per 1
$\mathrm{SD})$ based on the mean wall thickness at the infrarenal aorta. $^{30}$

Carotid intimal medial thickness (CIMT) has been extensively researched, likely due to its relative simplicity of measurement. While inter-rater reproducibility was a challenge with earlier studies, reported SDs have decreased in recent analysis, possibly associated with the advent of automated edge-tracking software. The decision of which portion of the carotid (common, bifurcation or internal) to evaluate is currently unsettled, although mean common carotid wall thickness has well-demonstrated accuracy and reproducibility. ${ }^{31}{ }^{32}$ CIMT has documented use as an endpoint in clinical intervention trials. $^{33} 34$ Additionally, meta-analysis of 37197 patients by Lorenz $e t a l^{55}$ showed that adjusted risk of future MI increases by about $10 \%$ for every $0.1 \mathrm{~mm}$ increase in CIMT, while the risk of future stroke increases by about $13 \%$ per $0.1 \mathrm{~mm}$ increase.

CT angiography enables imaging of the coronary arteries and assessment of plaque burden, both calcified and non-calcified. Comparison between coronary CT and intravascular ultrasound (IVUS) via meta-analysis has shown high sensitivity and specificity for coronary disease, suggesting that coronary CT is likely an accurate proxy for the invasive gold-standard test. ${ }^{36}$ While IVUS is unable to evaluate the adventitia, coronary CT evaluates intraluminal plaque, media and adventitia. In addition, CT angiography has been compared against invasive coronary angiography; this has demonstrated high sensitivity and specificity for detection of clinically significant lesions, with negative predictive value of $>90 \%{ }^{37-39}$ Additionally, both obstructive and non-obstructive diseases on CT angiography have been demonstrated in a prospective cohort to significantly associate with mortality with risk-adjusted HRs of 2.6 and 1.6, respectively. ${ }^{40}$

Coronary artery calcium (CAC) score can also be determined from coronary CT. CAC score is typically calculated by the Agatston method, in which the total area of coronary calcification in $\mathrm{mm}^{2}$ is multiplied by the maximum attenuation in Hounsfield units. ${ }^{41}$ In general, calcification is thought to represent a relatively late stage of the atherosclerotic process. Calcification on coronary CT is well correlated with calcification on postmortem histological analysis. ${ }^{42}$ CAC score has also been linked to cardiovascular events; one study showed a relative risk of 9.6 for CAC score $>100 \mathrm{vs}<100$, while the absence of CAC confers a very low risk of future cardiovascular events. ${ }^{43}$ Some data have also suggested that coronary calcification density may be inversely correlated with cardiovascular events. $^{44}$

IVUS allows for the identification and classification of plaques into collagen, fibrolipid, calcium or calcified necrotic using spectral analysis. This method has been validated against pathological specimens with $67 \%-92 \%$ accuracy, depending on the type of lesion. ${ }^{45}$ In patients undergoing imaging for acute coronary syndrome, thin-capped fibroatheromas (TCFAs) identified on IVUS are the highest risk category of plaque; 
however, this corresponds to a relatively low event rate of $4.9 \%$ at 3.4 years in the Providing Regional Observations to Study Predictors of Events in the Coronary Tree (PROSPECT) study (vs an event rate of $9.6 \%$ for $>70 \%$ occlusion and a $20.4 \%$ overall rate of repeat events) ${ }^{46}$ Serial imaging studies at 1 year demonstrate that $75 \%$ of TCFAs identified at baseline have 'healed' at 1 year, while TCFAs developed in new locations. ${ }^{47}$ Interestingly, in the European Collaborative Project on Inflammation and Vascular Wall Remodeling in Atherosclerosis - Intravascular Ultrasound (ATHEROREMO-IVUS) study patients with TCFA and plaque burden $>70 \%$ were at increased risk of Major Adverse Cardiovascular Events (MACE) at 6 months and at 1 year, while those with TCFA and $<70 \%$ plaque burden were only at increased risk at 1 year. This may be related to the relatively small sample (581 patients, $7.8 \%$ with MACE at 1 year), but could also be due to temporal effects of atherosclerotic progression. ${ }^{48}$

While prior pathological studies have shown an ordinal progression in the development of atherosclerosis, the progression of an individual lesion from fatty streak to TCFA is not inevitable. In fact, IVUS follow-up at 1 year showed that $75 \%$ of TCFAs 'healed', or became thickcapped fibroatheromas at 1 year. ${ }^{47}$ Given our present knowledge of the pathophysiology of atherogenesis, it seems likely that the utility of a particular imaging modality may be dependent on which stage of atherogenesis a particular lesion is at. For example, it is unlikely that calcified lesions would have high inflammatory activity, yet both types of lesions are clinically significant. Growth of a plaque may not increase inflammation on PET/CT, but could lead to evident plaque development on coronary CT, ultrasound or MRI.

Optical coherence tomography (OCT) is a second intravascular imaging technique that can be used to obtain high-resolution images that reliably differentiate coronary plaques into either fibrous plaque, fibrocalcific plaque or lipid-rich plaque, with good interobserver and intraobserver reliability, and with good correlation to histology. ${ }^{49}$ Furthermore in clinical research settings, OCT may be considered to demonstrate advanced stages of atherosclerosis, since calcification and lipid-rich histology are late events in Stary's model. Of note, patients undergoing percutaneous coronary intervention (PCI) who had lipidrich plaque in non-culprit regions (culprit considered previous PCI site) had significantly greater incidence of non-culprit major adverse cardiac events after a mean of 26 months of follow-up (7.2\% incidence rate in those with lipid-rich plaque, vs $2.6 \%$ in those without). ${ }^{50}$

\section{Summary}

With the exception of IVUS and OCT, these techniques are all non-invasive. Given that they represent anatomical changes in the vasculature, they would have relatively greater utility in detecting changes to moderate, but not yet clinically significant, disease. Further investigation is needed to validate the comparative sensitivity of these modalities. Additionally, it is possible that specific treatment effects may be more amenable to measurement by some modalities rather than others. For example, treatment with antihypertensives may decrease the shear stress, thereby decreasing plaque growth measured anatomically. Treatment with anti-inflammatory agents, such as in the recent CANTOS, would likely decrease vascular inflammation as measured by PET/CT. Treatment with statins, which both improve the lipid profile and inflammatory profile, may improve vascular disease on multiple modalities. Indeed, plaque regression on IVUS has been previously demonstrated after treatment with high-intensity statins in the Reversal of Atherosclerosis with Aggressive Lipid Lowering (REVERSAL) and A Study to Evaluate the Effect of Rosuvastatin on Intravascular Ultrasound-Derived Coronary Atheroma Burden (ASTEROID) trials. $^{51}{ }^{52}$ Further investigation using multiple imaging modalities concurrently over time is needed to determine which modalities provide the most incremental value at any given points in the development of atherosclerosis.

\section{CONCLUSION}

In conclusion, in order to decrease the burden of CVD, we must treat clinical CVD and prevent subclinical disease from leading to clinical disease. In order to identify and understand subclinical disease, utilisation of various imaging modalities and their appropriateness in the ordinal progression of atherosclerosis may be highly beneficial to speed understanding of therapies potentially used for treatment of CVD.

Acknowledgements This research was made possible through the NIH Medical Research Scholars Program, a public-private partnership supported jointly by the $\mathrm{NIH}$, and the generous contributions to the Foundation for the $\mathrm{NIH}$ from the Doris Duke Charitable Foundation (DDCF Grant \#2014194), Genentech, Elsevier and other private donors.

Contributors The idea for this review was conceived by JWG and NNM. The work was planned and executed by JWG under the supervision of NNM.

Funding NNM's lab is supported by the National Heart, Lung, and Blood Institute Intramural Program (HL006193-02), National Institutes of Health. JWG is supported by the Doris Duke Charitable Foundation (2014194).

Competing interests NNM is a full-time US government employee and receives research grants through the NHLBI from AbbVie, Janssen, Celgene and Novartis.

Patient consent Not required.

Provenance and peer review Not commissioned; externally peer reviewed.

Data sharing statement There are no unpublished data for this review.

Open access This is an open access article distributed in accordance with the Creative Commons Attribution Non Commercial (CC BY-NC 4.0) license, which permits others to distribute, remix, adapt, build upon this work non-commercially, and license their derivative works on different terms, provided the original work is properly cited, appropriate credit is given, any changes made indicated, and the use is non-commercial. See: http://creativecommons.org/licenses/by-nc/4.0/.

\section{REFERENCES}

1. Stary HC, Chandler AB, Dinsmore RE, et al. A definition of advanced types of atherosclerotic lesions and a histological classification of atherosclerosis. A report from the committee on vascular lesions of the council on arteriosclerosis, American Heart Association. Circulation 1995;92:1355-74. 
2. Libby P, Ridker PM, Hansson GK. Progress and challenges in translating the biology of atherosclerosis. Nature 2011;473:317-25

3. Burke AP, Farb A, Malcom GT, et al. Coronary risk factors and plaque morphology in men with coronary disease who died suddenly. $N$ Engl J Med 1997;336:1276-82.

4. Sugiyama T, Yamamoto E, Bryniarski K, et al. Nonculprit plaque characteristics in patients with acute coronary syndrome caused by plaque erosion vs plaque rupture: a 3-vessel optical coherence tomography study. JAMA Cardiol 2018;3:207-214

5. Barton P, Andronis L, Briggs A, et al. Effectiveness and cost effectiveness of cardiovascular disease prevention in whole populations: modelling study. BMJ 2011;343:d4044.

6. Raikou M, McGuire A, Colhoun HM, et al. Cost-effectiveness of primary prevention of cardiovascular disease with atorvastatin in type 2 diabetes: results from the Collaborative Atorvastatin Diabetes Study (CARDS). Diabetologia 2007;50:733-.

7. Goff DC, Lloyd-Jones DM, Bennett G, et al. 2013 ACC/AHA guideline on the assessment of cardiovascular risk: a report of the American College of Cardiology/American Heart Association Task Force on Practice Guidelines. Circulation 2014;129:S49-73.

8. Takx RA, Partovi S, Ghoshhajra BB. Imaging of atherosclerosis. Int $J$ Cardiovasc Imaging 2016;32:5-12.

9. Joshi FR, Lindsay AC, Obaid DR, et al. Non-invasive imaging of atherosclerosis. Eur Heart J Cardiovasc Imaging 2012;13:205-18.

10. Vancraeynest $D$, Pasquet $A$, Roelants $V$, et al. Imaging the vulnerable plaque. J Am Coll Cardiol 2011;57:1961-79.

11. Verjans JW, Jaffer FA. Biological imaging of atherosclerosis: moving beyond anatomy. J Cardiovasc Transl Res 2013;6:681-94.

12. Maurovich-Horvat P, Ferencik M, Voros S, et al. Comprehensive plaque assessment by coronary CT angiography. Nat Rev Cardiol 2014;11:390-402.

13. Harris RA, Nishiyama SK, Wray DW, et al. Ultrasound assessment of flow-mediated dilation. Hypertension 2010;55:1075-85.

14. Matsuzawa Y, Kwon TG, Lennon RJ, et al. Prognostic Value of FlowMediated Vasodilation in Brachial Artery and Fingertip Artery for Cardiovascular Events: A Systematic Review and Meta-Analysis. $J$ Am Heart Assoc 2015;4:e002270.

15. Corretti MC, Anderson TJ, Benjamin EJ, et al. Guidelines for the ultrasound assessment of endothelial-dependent flow-mediated vasodilation of the brachial artery: a report of the International Brachial Artery Reactivity Task Force. J Am Coll Cardiol 2002;39:257-65

16. Charakida M, de Groot E, Loukogeorgakis SP, et al. Variability and reproducibility of flow-mediated dilatation in a multicentre clinical trial. Eur Heart J 2013;34:3501-7.

17. Bonetti PO, Pumper GM, Higano ST, et al. Noninvasive identification of patients with early coronary atherosclerosis by assessment of digital reactive hyperemia. J Am Coll Cardiol 2004;44:2137-41.

18. Tarkin JM, Joshi FR, Rudd JH. PET imaging of inflammation in atherosclerosis. Nat Rev Cardiol 2014;11:443-57.

19. Rudd JH, Warburton EA, Fryer TD, et al. Imaging atherosclerotic plaque inflammation with [18F]-fluorodeoxyglucose positron emission tomography. Circulation 2002;105:2708-11.

20. Tawakol A, Fayad ZA, Mogg R, et al. Intensification of statin therapy results in a rapid reduction in atherosclerotic inflammation: results of a multicenter fluorodeoxyglucose-positron emission tomography/ computed tomography feasibility study. J Am Coll Cardiol 2013;62:909-17.

21. Figueroa AL, Abdelbaky A, Truong QA, et al. Measurement of arterial activity on routine FDG PET/CT images improves prediction of risk of future CV events. JACC Cardiovasc Imaging 2013;6:1250-9.

22. Tanaka H, Munakata M, Kawano $\mathrm{Y}$, et al. Comparison between carotid-femoral and brachial-ankle pulse wave velocity as measures of arterial stiffness. J Hypertens 2009;27:2022-7.

23. Mitchell GF, Parise H, Benjamin EJ, et al. Changes in arterial stiffness and wave reflection with advancing age in healthy men and women: the Framingham Heart Study. Hypertension 2004:43:1239-45.

24. Ben-Shlomo Y, Spears M, Boustred C, et al. Aortic pulse wave velocity improves cardiovascular event prediction: an individual participant meta-analysis of prospective observational data from 17,635 subjects. J Am Coll Cardiol 2014;63:636-46.

25. Mitchell GF, Hwang SJ, Vasan RS, et al. Arterial stiffness and cardiovascular events: the Framingham Heart Study. Circulation 2010;121:505-11.

26. Vlachopoulos C, Aznaouridis K, Stefanadis C. Prediction of cardiovascular events and all-cause mortality with arterial stiffness. $J$ Am Coll Cardiol 2010;55:1318-27.

27. Redheuil A, Wu CO, Kachenoura N, et al. Proximal aortic distensibility is an independent predictor of all-cause mortality and incident CV events: the MESA study. J Am Coll Cardiol 2014;64:2619-29.
28. Criqui $\mathrm{MH}$, Langer RD, Fronek $\mathrm{A}$, et al. Mortality over a period of 10 years in patients with peripheral arterial disease. $N$ Engl $J$ Med 1992;326:381-6.

29. Malayeri AA, Natori S, Bahrami $\mathrm{H}$, et al. Relation of aortic wall thickness and distensibility to cardiovascular risk factors (from the Multi-Ethnic Study of Atherosclerosis [MESA]). Am J Cardiol 2008:102:491-6.

30. Maroules CD, Rosero E, Ayers C, et al. Abdominal aortic atherosclerosis at MR imaging is associated with cardiovascular events: the Dallas heart study. Radiology 2013;269:84-91.

31. Naqvi TZ, Lee MS. Carotid intima-media thickness and plaque in cardiovascular risk assessment. JACC Cardiovasc Imaging 2014;7:1025-38

32. Pignoli P, Tremoli E, Poli A, et al. Intimal plus medial thickness of the arterial wall: a direct measurement with ultrasound imaging. Circulation 1986;74:1399-406.

33. Kastelein JJ, Akdim F, Stroes ES, et al. Simvastatin with or without ezetimibe in familial hypercholesterolemia. N Engl J Med 2008;358:1431-43.

34. Kastelein JJ, van Leuven SI, Burgess L, et al. Effect of torcetrapib on carotid atherosclerosis in familial hypercholesterolemia. $N$ Engl $J$ Med 2007;356:1620-30.

35. Lorenz MW, Markus HS, Bots ML, et al. Prediction of clinical cardiovascular events with carotid intima-media thickness: a systematic review and meta-analysis. Circulation 2007:115:459-67.

36. Fischer $\mathrm{C}$, Hulten $\mathrm{E}$, Belur $\mathrm{P}$, et al. Coronary $\mathrm{CT}$ angiography versus intravascular ultrasound for estimation of coronary stenosis and atherosclerotic plaque burden: a meta-analysis. J Cardiovasc Comput Tomogr 2013;7:256-66.

37. Meijboom WB, Meijs MF, Schuijf JD, et al. Diagnostic accuracy of 64-slice computed tomography coronary angiography: a prospective, multicenter, multivendor study. J Am Coll Cardiol 2008:52:2135-44.

38. Miller JM, Rochitte CE, Dewey M, et al. Diagnostic performance of coronary angiography by 64 -row CT. N Engl J Med 2008;359:2324-36.

39. Budoff MJ, Dowe D, Jollis JG, et al. Diagnostic performance of 64-multidetector row coronary computed tomographic angiography for evaluation of coronary artery stenosis in individuals without known coronary artery disease: results from the prospective multicenter ACCURACY (Assessment by Coronary Computed Tomographic Angiography of Individuals Undergoing Invasive Coronary Angiography) trial. J Am Coll Cardiol 2008;52:1724-32.

40. Min JK, Dunning A, Lin FY, et al. Age- and sex-related differences in all-cause mortality risk based on coronary computed tomography angiography findings results from the International Multicenter CONFIRM (Coronary CT Angiography Evaluation for Clinical Outcomes: An International Multicenter Registry) of 23,854 patients without known coronary artery disease. J Am Coll Cardiol 2011;58:849-60.

41. Agatston AS, Janowitz WR, Hildner FJ, et al. Quantification of coronary artery calcium using ultrafast computed tomography. J Am Coll Cardiol 1990;15:827-32.

42. Rumberger JA, Simons DB, Fitzpatrick LA, et al. Coronary artery calcium area by electron-beam computed tomography and coronary atherosclerotic plaque area. A histopathologic correlative study. Circulation 1995;92:2157-62.

43. Arad $\mathrm{Y}$, Goodman KJ, Roth M, et al. Coronary calcification, coronary disease risk factors, C-reactive protein, and atherosclerotic cardiovascular disease events: the St. Francis Heart Study. J Am Coll Cardiol 2005;46:158-65.

44. Criqui $\mathrm{MH}$, Denenberg JO, Ix JH, et al. Calcium density of coronary artery plaque and risk of incident cardiovascular events. JAMA 2014;311:271-8

45. Nair A, Kuban BD, Tuzcu EM, et al. Coronary plaque classification with intravascular ultrasound radiofrequency data analysis. Circulation 2002;106:2200-6.

46. Stone GW, Maehara A, Lansky AJ, et al. A prospective naturalhistory study of coronary atherosclerosis. N Engl J Med 2011;364:226-35.

47. Kubo T, Maehara A, Mintz GS, et al. The dynamic nature of coronary artery lesion morphology assessed by serial virtual histology intravascular ultrasound tissue characterization. J Am Coll Cardiol 2010;55:1590-7.

48. Cheng JM, Garcia-Garcia HM, de Boer SP, et al. In vivo detection of high-risk coronary plaques by radiofrequency intravascular ultrasound and cardiovascular outcome: results of the ATHEROREMO-IVUS study. Eur Heart J 2014;35:639-47.

49. Yabushita H, Bouma BE, Houser SL, et al. Characterization of human atherosclerosis by optical coherence tomography. Circulation 2002;106:1640-5. 
50. Xing L, Higuma T, Wang Z, et al. Clinical significance of lipid-rich plaque detected by optical coherence tomography: a 4-year followup study. J Am Coll Cardiol 2017;69:2502-13.

51. Nissen SE, Nicholls SJ, Sipahi I, et al. Effect of very high-intensity statin therapy on regression of coronary atherosclerosis: the ASTEROID trial. JAMA 2006;295:1556-65.
52. Nissen SE, Tuzcu EM, Schoenhagen P, et al. Effect of intensive compared with moderate lipid-lowering therapy on progression of coronary atherosclerosis: a randomized controlled trial. JAMA 2004;291:1071-80. 